\title{
RECHERCHES \\ SUR LA RÉSISTANCE DE LA PERCHE-SOLEIL (Eupomotis gibbosus L.) AUX VARIATIONS DE LA SALINITÉ
}

\author{
par M. SECONDAT \\ Docteur es sciences. \\ Chargé de la direction de la Station d'Hydrobiologie et de Pisciculture \\ de l'École Nationale Supérieure Agronomique de Toulouse.
}

Une note publiée en 1919 (1) signalait qu'une Perche-Soleil avait été capturée dans les eaux du grand étang de Vaccarès. La présence de ce poisson en eau saumâtre nous ayant paru fort intéressante, nous avons entrepris l'étude expérimentale de sa résistance à la salinité.

Ces premières investigations avaient pour but de déterminer dans quelle mesure cette espèce supporte l'eau salée et d'observer son comportement dans ce milieu.

Nos animaux d'expérience, d'une longueur de 4 à 5 centimètres, provenaient d'un étang de la banlieue toulousaine. Après un jeûne de plusieurs jours ces poissons étaient placés dans des cristallisoirs renfermant chacun deux litres d'une solution de chlorure de sodium pur dans de l'eau de source titrant 16 à 17 degrés hydrotimétriques et de $\mathrm{pH}$ : 7,8-8. Leurs besoins respiratoires étaient assurés par un barbotage d'air; enfin, pour maintenir une température constante, les récipients étaient plongés dans un grand bac à circulation d'eau permanente formant thermostat.

Dans une première série d'expériences, les sujets, prélevés en eau douce, étaient brusquement immergés dans le milieu salin. De telles conditions nous ont permis de faire les remarques suivantes :

Dans des solutions de faible concentration, dont nous préciserons plus loin la limite supérieure, la réaction du poisson qui se manifeste dès l'immersion en eau salée, se borne à une courte mais violente agitation, après laquelle le sujet reprend son état normal. Mais, lorsqu'on utilise des milieux de concentration plus élevée, le poisson accuse immédiatement

(1) Cabanes (G.). - Un poisson nouveau pour notre faune méridionale (Eupomotis gibbosus L.). Annales du Musée d'Histoire Naturelle de Marseille. 1919, t. XVII, pp. 49-51. 
des symptômes bien caractérisés qui cessent de se manifester aussitôt que l'animal est replacé dans l'eau douce, mais qui se prolongent plus ou moins suivant les individus, quand on les laisse dans la solution. La crise terminée, le sujet retrouve son état normal et le conserve parfois pendant plusieurs heures.

Voici la relation détaillée d'une observation :

Le 24 juillet, à $16 \mathrm{~h}$. 25, une Perche-Soleil prélevée en eau douce est immergée dans une solution de chlorure de sodium à $15 \%$, la température de l'eau est de 2205 . Dès l'entrée en eau salée, le poisson est en proie a une agitation violente qui se prolonge pendant 10 secondes, puis il s'immobilise en surface, couché sur le flanc, dans un état comateux.

A $16 \mathrm{~h}$. 30, le poisson nage très rapidement, incliné sur le côté en suivant les parois du cristallisoir; après quelques secondes il s'immobilise de nouveau en surface et ses battements operculaires se ralentissent.

A 16 h. 40, même état d'immobilité, le rythme respiratoire est très faible, le sujet ne réagit plus au toucher.

A 16 h. 50, tendance à l'amélioration, la Perche-Soleil se redresse lentement et reprend sa station normale; puis, elle descend au fond du récipient et nage sans vigueur. C'est ensuite un retour à la surface de quelques secondes suivi d'une nouvelle descente au fond. normal.

A $17 \mathrm{~h}$. 05, le poisson semble avoir retrouvé son comportement

Le lendemain matin, à 7 heures, la Perche-Soleil est trouvée morte, la bouche largement ouverte, les opercules soulevés.

Les résultats de nos expériences réalisées dans des solutions de concentrations différentes et à des températures variables, sont résumés dans le tableau $I$.

L'examen de ces résultats nous permet de dégager les observations suivantes :

$1^{0}$ Dès le début de l'immersion en eau salée, tous les sujets d'expériences accusent une courte, mais violente phase d'agitation, dont la durée semble variable avec les individus;

$2^{\circ}$ Les états comateux successifs qui se manifestent ensuite ne se produisent que dans les solutions salines dont la concentration est au moins égale à $12 \%$. Toutefois, la durée de ces symptômes augmente avec le titre des solutions.

$3^{0}$ Le temps de survie en eau salée s'abaisse évidemment quand la concentration de la solution augmente, mais pour une salinité déterminée du milieu ambiant, la résistance de la Perche-Soleil diminue nettement lorsque la température s'élève.

Nous avons recherché ensuite jusqu'à quel degré de salinité on peut faire vivre la Perche-Soleil, quand on augmente progressivement la concentration du milieu en chlorure de sodium. 
Tableau I.

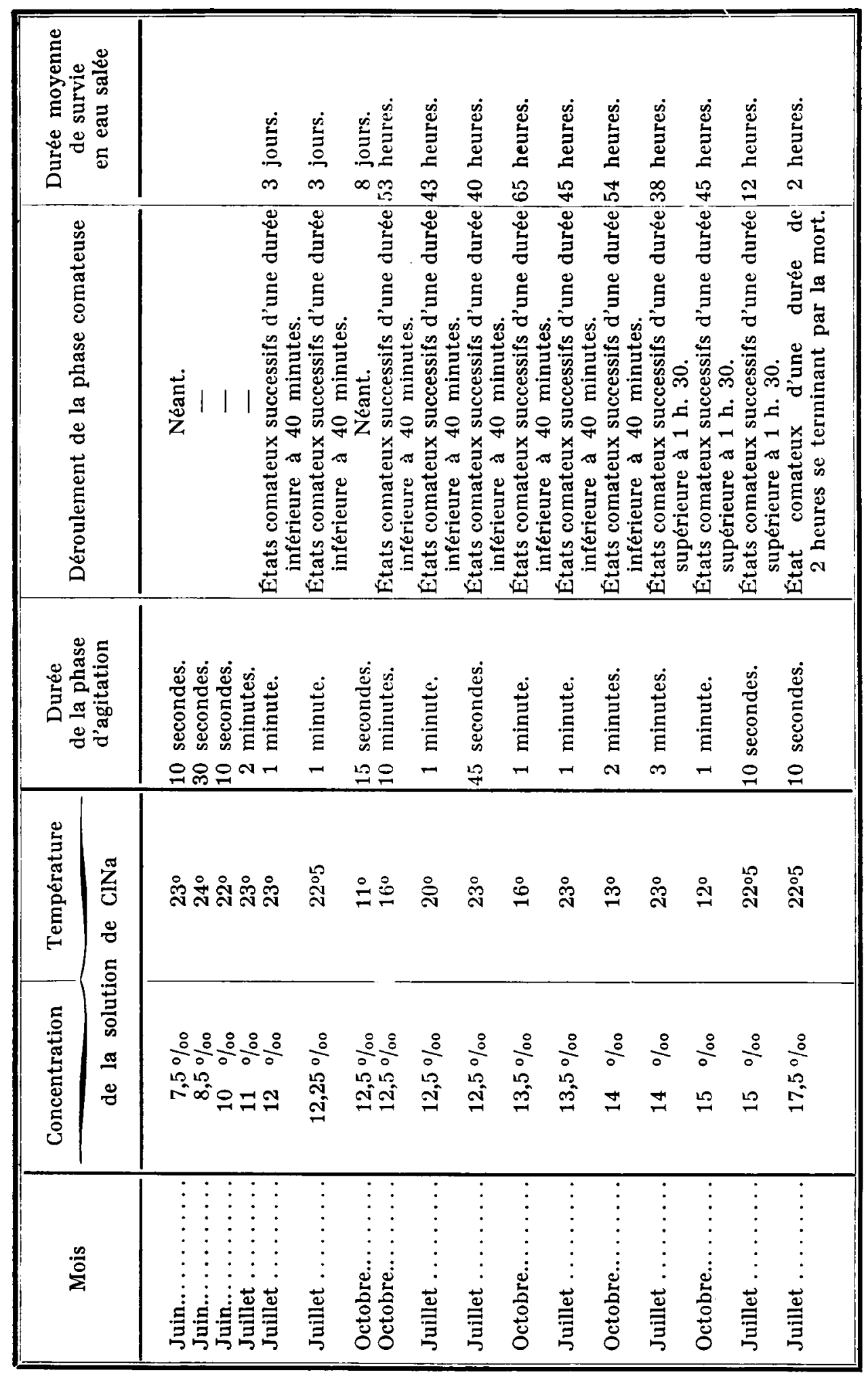


Dans ce but, les poissons d'expériences étaient plongés dans des cristallisoirs renfermant chacun une solution de faible concentration, dont le titre s'élevait lentement sous le seul effet de l'évaporation à l'air libre. Les résultats obtenus figurent dans le tableau II.

Tableau II. - Température de la solution : $24^{\circ}-21^{\circ}$.

\begin{tabular}{|c|c|c|}
\hline $\begin{array}{c}\text { Jours } \\
\text { d'expérience }\end{array}$ & $\begin{array}{l}\text { Concentration } \\
\text { de la solution de } \mathrm{ClNa}\end{array}$ & $\begin{array}{l}\text { Comportement } \\
\text { des poissons }\end{array}$ \\
\hline 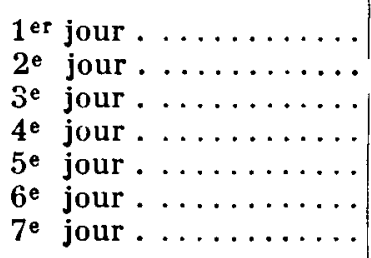 & $\begin{array}{r}8 \% \\
10 \% \\
12 \% \\
13 \% \\
14 \% \\
14,8 \% \\
15,6 \%\end{array}$ & $\begin{array}{c}\text { Normal } \\
\text { Normal } \\
\text { Normal } \\
\text { Normal } \\
\text { Troubles de l'équilibre } \\
\text { Troubles de l'équilibre } \\
\text { Mort des poissons }\end{array}$ \\
\hline
\end{tabular}

Cet essai d'adaptation lente montre donc que la Perche-Soleil immergée dans une solution de $\mathrm{Cl} \mathrm{Na} \mathrm{à} 8 \%$, à la température de $24^{\circ}-21^{\circ}$ meurt au bout de sept jours, alors que la concentration du milieu, augmentant sous l'effet de l'évaporation s'élève à $15,6 \%$ de chlorure de sodium.

Si nous comparons maintenant le comportement des sujets soumis à des changements brusques de salinité, avec celui des poissons exposés à des variations lentes, nous constatons que la résistance de la PercheSoleil augmente, lorsqu'on accroît progressivement la concentration saline du milieu ambiant.

Néanmoins, il est intéressant de remarquer qu'à température égale, les symptômes d'intoxication manifestés par les poissons sont beaucoup moins accentués et apparaissent pour des concentrations salines un peu plus élevées, lorsque les sujets d'expérience sont exposés à des variations lentes de salinité.

La Perche-Soleil semble donc douée d'un certain degré d'euryhalinité ; cependant, sa résistance à la salinité diminue sensiblement avec l'élévation de la température du milieu ambiant. 